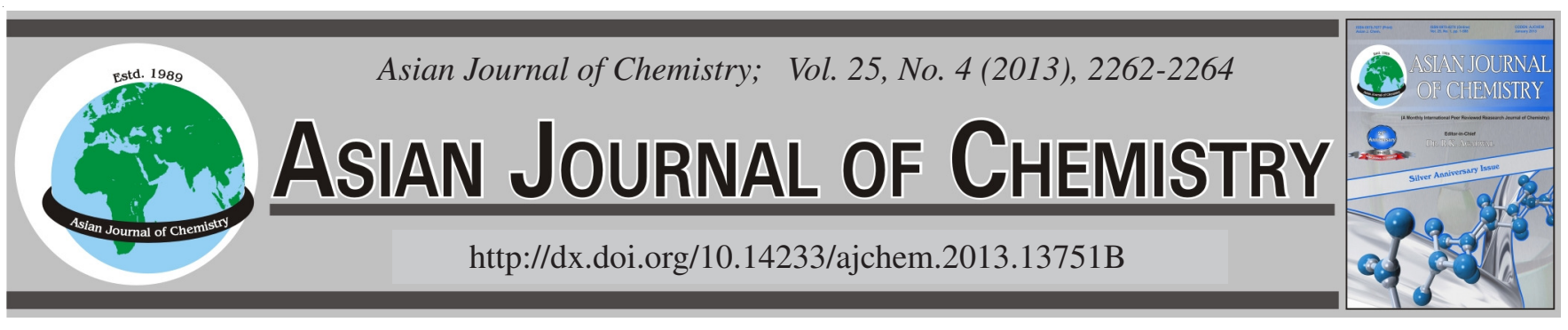

\title{
Radiation-Induced Graft Copolymerization of Acrylamide onto Carboxymethylcellulose and Modification of its Chemical Structure
}

\section{Fatemeh Soleimani ${ }^{1,}$, Mohammad Sadeghi ${ }^{2}$, Hadis Shasevari ${ }^{1}$, Arezoo Soleimani ${ }^{1}$ and Hossein SadeGhi ${ }^{3}$}

\author{
${ }^{1}$ Young Researchers Club, Khorramabad Branch, Islamic Azad University, Khorramabd, Iran \\ ${ }^{2}$ Department of Chemistry, Science Faculty, Arak Branch, Islamic Azad University, Arak, Iran \\ ${ }^{3}$ Department of Chemistry, Science Faculty, Khorramabad Branch, Islamic Azad University, Khorramabad, Iran \\ *Corresponding author: Fax: +98 861 3670017; Tel: +98 916 1613256; E-mail: fatisoleymani@yahoo.com
}

\begin{abstract}
The monomer, acrylamide, was graft copolymerized onto carboxymethylcellulose using $\gamma$-rays as initiator. The reactions were carried out in a homogenous aqueous medium. After removal of the homopolymer, the graft copolymer was characterized by FTIR spectroscopy. The structure of pure pectin and grafted with monomer was also characterized by thermogravimetric analysis. The thermal properties of pure pectin and grafted with monomer were evaluated with a simultaneous thermal analysis system. The results showed that the thermal stability of grafted polyacrylamide samples was remarkably improved. A plausible mechanism of grafting has also been suggested. The overall activation energy for the grafting was estimated to be $36.60 \mathrm{~kJ} / \mathrm{mol}$.
\end{abstract}

Key Words: Carboxymethylcellulose, Acrylamide, $\gamma$-Radiation, Copolymerization.

\section{INTRODUCTION}

Graft copolymerization is an attractive means for modifying base polymers because grafting frequently results in the superposition of properties relating to the backbone and pendant chains. Considerable interest has been focused on chemical modification by free radical graft copolymerization of hydrophilic and hydrophobic vinyl monomers biopolymers such as polysaccharides ${ }^{1-5}$. These biodegradable and low cost graft copolymers, with new properties, can be used in many applications such as textiles, paper industry, agriculture, medical treatment and also in petroleum industry as flocculants and thickening agents ${ }^{6-8}$.

Graft copolymers are prepared by first generating free radicals on the polysaccharide backbone and then allowing these radicals to serve as macroinitiators for the vinyl monomers. Graft copolymerization can be carried out with different initiator systems. Among them, potassium persulfate, ammonium persulfate, benzoyl peroxide, azo bisisobutyronitrile and ceric ammonium nitrate are widely used for the synthesis of graft copolymers $^{9-11}$.

Radiation grafting technology is well established and accepted by industry. Radiation polymerization, radiation crosslinking and controlled degradation of polymers comprise most of commercial applications of radiation technology ${ }^{12}$.
The chosen polysaccharide for modification, i.e., carboxymethylcellulose (CMC), is the well-known and most important type of polysaccharide. Sodium salt of carboxymethylcellulose is the first water soluble ionic derivative of cellulose prepared in 1918 and produced commercially in the early 1920's in Germany. It has been the most important ionic cellulose ether with a worldwide annual production of 300,000 tons. It is widely used in pharmaceuticals, detergents, cosmetics, foods, paper and textile industries due to its viscosity-increasing and emulsifying properties. However, it may need to be further modified for some special applications.

Of the monomers grafted, acrylamide has been the most requently used one, mainly due to its highest grafting efficiency ${ }^{13-16}$, improving the thermal resistance of the graft copolymer $^{17}$ and also the subsequent alkaline hydrolysis of the grafting product to obtain water absorbents ${ }^{18,19}$. The present report describes graft copolymerization of acrylamide onto carboxymethylcellulose backbone, initiated by $\gamma$-rays.

\section{EXPERIMENTAL}

Carboxymethylcellulose sample (DS 0.52) was purched from Merch Co. Acrylamide (AAm, Fluka), was used after crystallization in acetone. All other chemicals were of analytical grade.

Preparation of graft copolymer: Carboxymethylcellulose solution was prepared in a one-liter reactor equipped with 
mechanical stirrer and gas inlet. Carboxymethylcellulose was dissolved in degassed distillated water. In general, $0.50 \mathrm{~g}$ of carboxymethylcellulose was dissolved in $30 \mathrm{~mL}$ of distillated degassed water. The reactor was placed in a water bath preset at $60{ }^{\circ} \mathrm{C}$. Then, variable amounts of acrylamide were added to the carboxymethylcellulose solution and the mixture was continuously stirred for $1 \mathrm{~h}$ under argon. The total volume of reaction was $40 \mathrm{~mL}$. The cold mixture was removed into a $250 \mathrm{~mL}$ aluminium tube. The inner wall of aluminium tube was covered with aluminium foil. The tube was closed tightly with the foil and paraffin film. The tube was then irradiated under $\gamma$-rays according to the desired total doses. Then, 500 $\mathrm{mL}$ methanol was added to the gelled product. After complete dewatering for $24 \mathrm{~h}$, the product was filtered, washed with fresh methanol $(2 \mathrm{~mL} \times 50 \mathrm{~mL})$ and dried at $50^{\circ} \mathrm{C}$.

Homopolymer extraction: The graft copolymer was freed from polyacrylamide homopolymer, by pouring $0.50 \mathrm{~g}$ of the product in $50 \mathrm{~mL}$ of dimethyl formamide solution. The mixture was stirred gently at room temperature for $48 \mathrm{~h}$. After complete removal of the homopolymer, the copolymer was filtered, washed with ethanol and dried in oven at $50{ }^{\circ} \mathrm{C}$ to reach a constant weight.

Infrared analysis: The samples were crushed with $\mathrm{KBr}$ to make pellets. Spectra were taken on an ABB Bomem MB100 FTIR spectrophotometer.

\section{RESULTS AND DISCUSSION}

Graft copolymerization mechanism: The mechanism of grafting acrylamide onto carboxymethylcellulose (CMC) using $\gamma$-rays as an initiator is shown in the Scheme-I. It should be mentioned that during the irradiation of acrylamide, carboxymethylcellulose and water ternary mixture, most of the energy is absorbed by water and only a very small fraction by other components. Thus, the initiation occurs mainly by an indirect effect. Hydroxyl radicals, formed during irradiation, add to one side of the AAm double bond and leads to the formation of an unpaired spin on the other side of the vinyl bond $^{21}$. In this way, homo polymerization of acrylamide is initiated. Attack of $\mathrm{OH}$ radicals on carboxymethylcellulose would lead almost solely to the break age of C-H bonds. This fact is very well known from radiation chemistry of alcohols and carbohydrates in aqueous solution. A much more probable pathway is the addition of a acrylamide molecule (not a radical) to the carboxymethylcellulose-based radical, followed by polymerization leading to the growth of a branched chain. The rate of grafting $\left(\mathrm{R}_{\mathrm{g}}\right)$ may be evaluated as measures of the rate of monomers disappearance by using the following expression ${ }^{18}$.

$$
\mathrm{R}_{\mathrm{g}}\left(\mathrm{mol} \mathrm{L}^{-1} \mathrm{~s}^{-1}\right)=1000 \mathrm{~W}_{3} / \mathrm{MTV}
$$

$\mathrm{M}\left(\mathrm{g} \mathrm{mol}^{-1}\right)$ is the molecular weight of the monomers. T and V stand for total reaction time $(\mathrm{s})$ and total volume $(\mathrm{mL})$ of the reaction mixture.

Overall activation energy of grafting $\left(\mathrm{E}_{\mathrm{a}}\right)$ may also be estimated from the temperature data through plotting $\ln \mathrm{R}_{\mathrm{g}}$ versus $1 / \mathrm{T}\left(\mathrm{K}^{-1}\right)$ for the initial portion of the data of the temperature series given in text. The slope of this Arrhenius plot (Fig. 1) resulted in a rough estimation of $E_{a}$ of grafting using the relationship slope $=-\mathrm{E}_{\mathrm{a}} / \mathrm{R}$; where $\mathrm{R}$ is the universal gas constant. Therefore, $\mathrm{E}_{\mathrm{a}}$ was found to be $36.60 \mathrm{~kJ} / \mathrm{mol}(8.75 \mathrm{kcal} / \mathrm{mol})$.

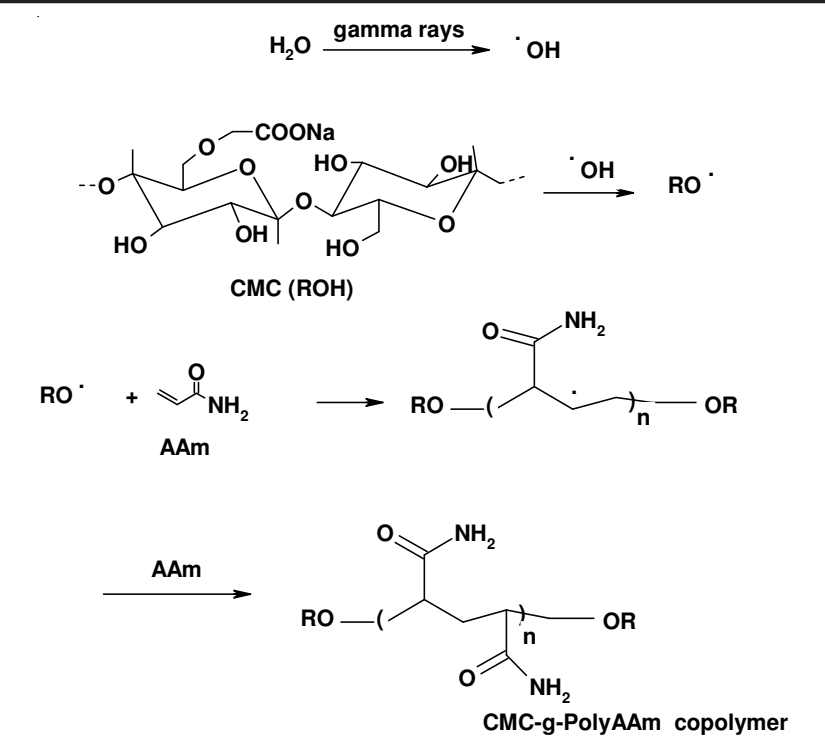

Scheme-I: A brief proposed mechanism for $\gamma$-rays-induced grafting of acrylamide onto carboxymethylcellulose

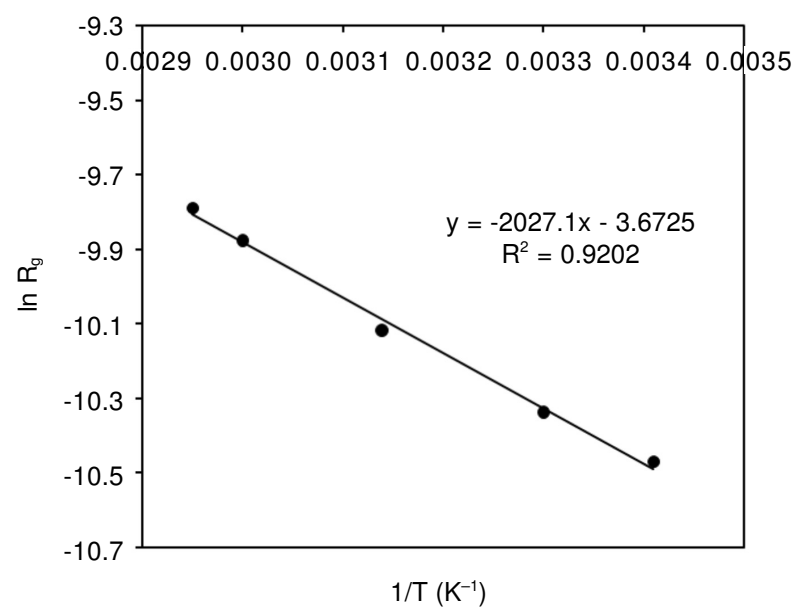

Fig. 1. Plot of $\ln \mathrm{R}_{\mathrm{g}}-1 / \mathrm{T}$ for estimating the activation energy of the graft polymerization reaction

FTIR spectroscopy: For identification of the graft copolymer, infrared spectroscopy was used. Fig. 2 shows the IR spectroscopy of CMC-g-PAAm copolymer. The graft copolymer product comprises a pure carboxymethylcellulose backbone with side chains that carry carboxamide functional groups that are evidenced by peaks at $1660 \mathrm{~cm}^{-1}$. In fact, In the spectrum of the graft copolymer (Fig. 1b), new peaks are appeared at 3206 and $1660 \mathrm{~cm}^{-1}$ that may be attributed to amide $\mathrm{NH}$ stretching, asymmetric and symmetric amide $\mathrm{NH}$ bending, respectively ${ }^{19}$.

Thermogravimetric analysis: TGA curves for carboxymethylcellulose and CMC-g-poly(acrylamide) copolymer are shown in Fig. 3. The grafted carboxymethylcellulose has shown improvement in thermal stability as clear from TGA curve. The initial decomposition temperature of the pectin on grafting was increased from $143-418{ }^{\circ} \mathrm{C}$ with maximum decomposition rate at $524{ }^{\circ} \mathrm{C}$, in comparison to original decomposition temperature of $316^{\circ} \mathrm{C}$ of carboxymethylcellulose. These observations have clearly indicated that grafting of CMC-g-poly(acrylamide copolymer has improved the thermal stability of carboxymethylcellulose ${ }^{20}$. 

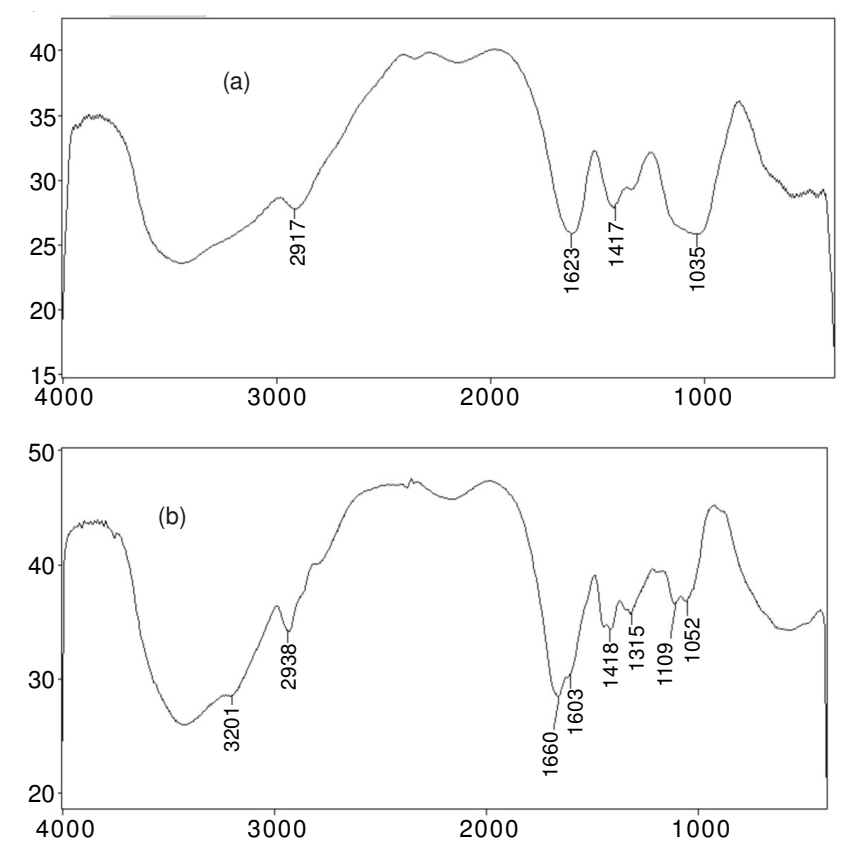

Fig. 2. FTIR spectra of (a) pure carboxymethylcellulose and (b) CMC-gPAAm copolymer

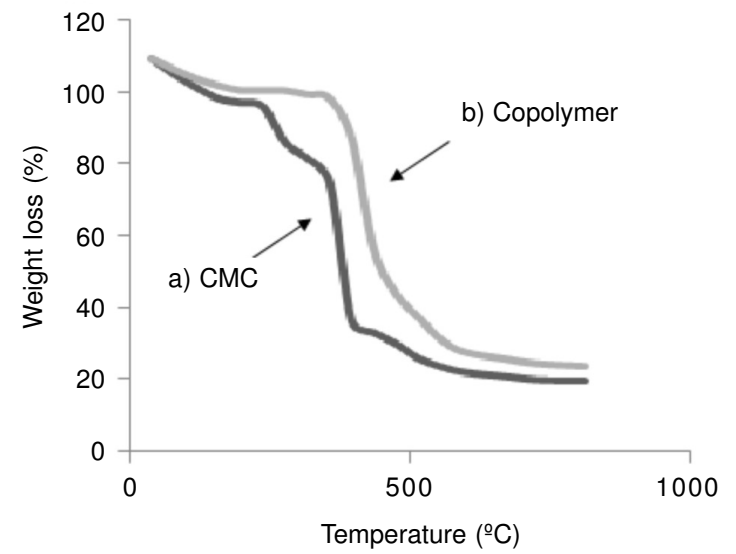

Fig. 3. TGA thermograms of (A) carboxymethylcellulose and (B) CMCg-poly(acrylamide) copolymer. Heating rate $20{ }^{\circ} \mathrm{C} / \mathrm{min}$, under $\mathrm{N}_{2}$

Solubility test: The simplest method to prove the formation of CMC-g-polyAAm is based on the solubility difference of the graft copolymer and the non-grafted homopolymer. Sucrose and polyAAm are soluble in water and DMF, respectively. When a reaction product was extracted with DMF and alternatively with water for $48 \mathrm{~h}$, an insoluble solid still remained. A physical mixture of carboxymethylcellulose and polyAAm was treated in the same way and was found to dissolve completely. Therefore, it is obvious that the resulted graft copolymer was not a simple physical mixture, but some chemical bonds must exist between the sucrose substrate and polyacrylamide macromolecules.

\section{Conclusion}

The polysaccharide, carboxymethylcellulose was graft copolymerized with synthetic monomer, acrylamide, using $\delta$-rays as efficient free radical initiators. In order to prove that monomer molecules were grafted, FTIR spectroscopy and TGA analysis were used. Empirical polymerization rate showed a first-order dependence on the monomers concentration and a half-order dependence on the initiator concentration. According to the slope of $\ln \mathrm{R}_{\mathrm{g}}$ versus $1 / \mathrm{T}$, the overall activation energy for graft copolymerization reaction was estimated to be $36.60 \mathrm{~kJ} / \mathrm{mol}$. In a previous work, we estimated similarly the overall activation energy to be $27.3 \mathrm{~kJ} / \mathrm{mol}$ for the grafting of acrylonitrile onto sucrose.

\section{REFERENCES}

1. F.L. Buchholz and G.T. Andrew, Modern Superabsorbent Polymer Technology, Wiley, New York (1997)

2. R. Po, J. Macromol. Sci.-Rev. Macromol. Chem. Phys., C34, 607 (1994).

3. N.A. Peppas and A.G. Mikes, In Hydrogels in Medicine and Pharmacy, CRC Press, Boca Raton, Florida, Vol. 1 (1986).

4. D. De Rossi, K. Kajiwara, Y. Osada and A. Yamauchi, Polymer Gels, Fundamentals and Biomedical Applications, Plenum, New York (1991).

5. K. Hogari, in ed.: F. Ashiya, Advances in Superabsorbent Polymers, American Chemical Society, Washington D.C. (1994).

6. K. Park, Controlled Drug Delivery; Challenges and Strategies, American Chemical Society, Washington, D.C (1997).

7. E. Rezai and R.R. Warner, J. Appl. Polym. Sci., 65, 1463 (1997).

8. K. Hitush and K. Shin, J. Appl. Polym. Sci., 64, 2259 (1997).

9. A. Pourjavadi, G.R. Mahdavinia and M.J. Zohuriaan-Mehr, J. Appl. Polym. Sci., 90, 3115 (2003).

10. F.L. Mi, C.Y. Kuan, S.S. Shyu, S.T. Lee and S.F. Chang, Carbohydr. Polym., 41, 389 (2000).

11. G.F. Fanta, In ed.: J.C. Salamone, In Polymeric Materials Encyclopedia, CRC Press (1987).

12. Y.J. Kim, K.J. Yoon and S.W. Ko, J. Appl. Polym. Sci., 78, 1797 (2000).

13. Y. Zhu, B. Pu, J. Zhang and J. Schen, J. Appl. Polym. Sci., 79, 572 (2001).

14. J.L. Willett, M.A. Kotnis, G.S. Obrin, G.F. Fanta and S.H. Gordan, J. Appl. Polym. Sci., 70, 1121 (1998).

15. D. Castel, A. Ricard and R. Audebert, J. Appl. Polym. Sci., 39, 11 (1990).

16. S. Kuwabara and H. Kubo, J. Appl. Polym. Sci., 60, 1965 (1996).

17. L.-M. Zhang and Y.-B. Tan, Macromol. Mater. Eng., 280-281, 59 (2000).

18. J. Branrup and E.H. Immergut, In Polymer Handbook, Wiley, New York, edn. 3 (1989).

19. R.M. Silverstein and F.X. Webster, Spectrometric Identification of Organic Compounds, Wiley, New York, edn. 6 (1998).

20. A. Pourjavadi and M.J. Zohuriaan-Mehr, J. Polym. Mater., 20, 1130 (2003).

21. H. Omidian, S.A. Hashemi, P.G. Sammes and I. Meldrum, Polymer, 39, 6697 (1998). 